\title{
A disautonomia cardíaca em pacientes com síndrome metabólica: uma revisão sistemática
}

Heart disautonomy in patients with metabolic syndrome: a systematic review

Desautonomía cardíaca en pacientes con síndrome metabólico: una revisión sistemática

Aline Fonseca de Oliveira Costa ${ }^{1}$, Thalita Thereza Menezes Carneiro ${ }^{1}$, Antônio da Silva Menezes Junior $^{1 *}$, Ana Luisa Adorno de Lima1.

\begin{abstract}
RESUMO
Objetivo: Construir uma revisão sistemática de literatura acerca das associações entre a variabilidade da frequência cardíaca e a síndrome metabólica. Métodos: Foram buscados artigos de forma sistemática nas bases de dados PubMed, Google Acadêmico, Lilacs e Cochrane, com os descritores "metabolic syndrome" AND "heart rate variability" AND "insulin resistance" Critérios de inclusão utilizados foram: 1. Estudo em humanos; 2. Pacientes acima de 18 anos; 3. Que possuam critérios diagnósticos de síndrome metabólica da OMS, NCEP-ATPIII ou IDF; 4. Com grupo controle; 5. Pacientes não possuírem outras comorbidades; 6 . Que correlacionem síndrome metabólica e variabilidade da frequência cardíaca. Idiomas escolhidos foram inglês, português e espanhol. Resultados: Oito estudos cumpriram os critérios de inclusão. Em pacientes com síndrome metabólica, a variabilidade da frequência cardíaca estava mais frequentemente reduzida, acentuando em mulheres e em pacientes com mais critérios diagnósticos, e está diretamente associada à resistência insulínica. Considerações finais: A variabilidade da frequência cardíaca é afetada de forma heterogênea entre homens e mulheres com síndrome metabólica. Além disso, a análise da VFC se mostrou uma ferramenta simples e eficaz que proporciona uma avaliação e identificação de comprometimentos da saúde e, por isso, estudos sobre o tema são de suma importância.
\end{abstract}

Palavras-chave: Variabilidade da frequência cardíaca, Síndrome metabólica, Resistência à insulina.

\begin{abstract}
Objective: To build a systematic literature review about the associations between heart rate variability and metabolic syndrome. Methods: Articles were systematically searched in the PubMed, Google Scholar, Lilacs and Cochrane databases, with the keywords "metabolic syndrome" AND "heart rate variability" AND "insulin resistance". Inclusion criteria used were: 1 . Study in humans; 2. Patients over 18 years old; 3. Who have diagnostic criteria for WHO metabolic syndrome, NCEP-ATPIII or IDF; 4. With control group; 5. Patients do not have other comorbidities; 6 . That correlate metabolic syndrome and heart rate variability. Languages chosen were English, Portuguese, and Spanish. Results: Eight studies met the inclusion criteria. In patients with metabolic syndrome, heart rate variability was more often reduced, accentuating in women and in patients with more diagnostic criteria, and is directly associated with insulin resistance. Final considerations: Heart rate variability is heterogeneously affected between men and women with metabolic syndrome. In addition, HRV analysis proved to be a simple and effective tool that provides an assessment and identification of health impairments and, therefore, studies on the topic are of paramount importance.
\end{abstract}

Key words: Heart rate variability, Metabolic syndrome, Insulin resistance.

\footnotetext{
${ }^{1}$ Pontifícia Universidade Católica de Goiás (PUC-GO), Goiânia - GO.

*E-mail: a.menezes.junior@uol.com.br
} 


\section{RESUMEN}

Objetivo: Construir una revisión bibliográfica sistemática sobre las asociaciones entre la variabilidad de la frecuencia cardíaca y el síndrome metabólico. Métodos: Se realizaron búsquedas sistemáticas de artículos en las bases de datos PubMed, Google Scholar, Lilacs y Cochrane, con las palabras clave "síndrome metabólico" Y "variabilidad de la frecuencia cardíaca" $Y$ "resistencia a la insulina". Los criterios de inclusión utilizados fueron: 1. Estudio en humanos; 2. Pacientes mayores de 18 años; 3. Que tengan criterios de diagnóstico para el síndrome metabólico de la OMS, NCEP-ATPIII o IDF; 4. Con grupo de control; 5 . Los pacientes no tienen otras comorbilidades; 6 . Que correlacionan el síndrome metabólico y la variabilidad de la frecuencia cardíaca. Los idiomas elegidos fueron inglés, portugués y español. Resultados: Ocho estudios cumplieron los criterios de inclusión. En pacientes con síndrome metabólico, la variabilidad de la frecuencia cardíaca se redujo con mayor frecuencia, acentuándose en mujeres y en pacientes con más criterios diagnósticos, y se asocia directamente con la resistencia a la insulina. Consideraciones finales: La variabilidad de la frecuencia cardíaca se ve afectada de manera heterogénea entre hombres y mujeres con síndrome metabólico. Además, el análisis de la VFC demostró ser una herramienta simple y eficaz que proporciona una evaluación e identificación de los problemas de salud y, por lo tanto, los estudios sobre el tema son de suma importancia.

Palabras clave: Variabilidad del ritmo cardíaco, Síndrome metabólico, Resistencia a la insulina.

\section{INTRODUÇÃO}

A disautonomia cardíaca é gerada por um desequilíbrio no Sistema Nervoso Autônomo (SNA). Dentre as diversas comorbidades que podem gerar a neuropatia autonômica, a Síndrome Metabólica (SM) tem sido destacada em diversos estudos atuais, uma vez que aumenta a mortalidade geral em cerca de 1,5 vezes e a cardiovascular em 2,5 vezes, além de se portar como um fator preditor para doença cardiovascular ou morte súbita cardíaca (CARVALHO MHC, et al., 2005; PONTES LM, et al., 2016).

A SM é uma doença crônica de origem multifatorial caracterizada por obesidade central, resistência à insulina, hiperglicemia, hipertensão e dislipidemia. Indivíduos com SM apresentam repercussões sistêmicas e predisposição principalmente à doenças cardiovasculares à complicações do diabetes mellitus tipo 2 (DM2). Visando comprovar e estabelecer uma relação entre a disautonomia cardíaca e a SM, estudos têm usado a Variabilidade da Frequência Cardíaca (VFC) para avaliar o sistema nervoso autônomo cardíaco e seu comportamento na presença de SM (RAMOS JS, et al., 2017; SILVA LR, et al., 2017).

A VFC é um marcador eficaz, não invasivo e de boa sensibilidade para avaliar a atividade autonômica cardíaca. Seu mecanismo consiste em descrever as oscilações no intervalo entre batimentos cardíacos consecutivos (intervalos R-R), assim como oscilações entre frequências cardíacas instantâneas consecutivas, o que permite avaliar a modulação da variabilidade cardíaca pelo SNA. Assim, através de uma análise espectral da VFC pode-se inferir se o indivíduo está propenso a apresentar ou se já apresenta alterações do sistema autônomo (CAMBRI LT, et al., 2009; VANDERLEI LCM, et al., 2009).

Diversos estudos transversais analisaram a relação entre SM e VFC ao estabelecerem grupos controle sem a presença de SM (SM-) e grupos portadores de SM (SM+), além de excluírem fatores interferentes na pesquisa (drogas em uso, tabagismo, alcoolismo, dentre outros hábitos de vida). Os estudos já publicados, em sua maioria, utilizam alguma das definições de SM mais aceitas por sociedades reconhecidas como a Organização Mundial de Saúde (OMS), National Cholesterol Education Program's Adult Treatment Panel III (NCEAP-ATPIII) ou International Diabetes Federation (IDF). Alguns estudos, no entanto, utilizam diferentes definições de SM, protocolos de coletas e de análise de dados, prejudicando a análise e interpretação dos resultados, gerando prejuízo a este estudo. O objetivo desta pesquisa, portanto, foi verificar a existência da relação entre VFC e SM. 


\section{MÉTODOS}

Este é um estudo do tipo revisão sistemática por meio de consulta às bases online (PubMed) National Library of Medicine, Cochrane Library, Literatura Latino-Americana e do Caribe em Ciências da Saúde (LILACS) e Google Acadêmico (neste, a partir de 2015, com os descritores: "metabolic syndrome" AND "heart rate variability" AND "insuline resistance".

Dois pesquisadores avaliaram de forma separada e independente, fazendo primeiramente a leitura dos títulos e seus resumos (quando disponíveis) para avaliar a elegibilidade dos artigos. Quando houve uma maior divergência de opinião entre os pesquisadores, foi adicionada a opinião de um terceiro pesquisador.

Critérios de inclusão: ser realizado em humanos, ter como população de observação pessoas acima de 18 anos, utilizar como critério de SM os parâmetros estabelecidos por uma das seguintes instituições OMS, NCEP-ATPIII ou IDF, possuir um grupo controle, a população em estudo não possuir outras comorbidades crônicas tais como esclerose múltipla, doença renal crônica, problemas cardíacos e correlacionar os parâmetros da VFC com a SM entre pacientes SM- e SM+ (Quadro 1).

Quadro 1 - Parâmetros da variabilidade da frequência cardíaca.

\begin{tabular}{|c|c|c|}
\hline Abreviações & Parâmetros da VFC & Representação no SNA \\
\hline \multicolumn{3}{|c|}{ Parâmetros do domínio do tempo } \\
\hline SDNN & $\begin{array}{c}\text { Desvio padrão dos intervalos } \\
\text { R-R normais }\end{array}$ & $\begin{array}{l}\text { Representa as atividades } \\
\text { simpática e parassimpática }\end{array}$ \\
\hline RMSSD & $\begin{array}{l}\text { Raiz quadrada média das } \\
\text { diferenças sucessivas }\end{array}$ & $\begin{array}{l}\text { Representa atividade } \\
\text { parassimpática }\end{array}$ \\
\hline \multicolumn{3}{|c|}{ Parâmetros do domínio da frequência } \\
\hline VLF & $\begin{array}{l}\text { Potência da frequência muito } \\
\text { baixa }(0,003-0,04 \mathrm{~Hz})\end{array}$ & Funcionamento das \\
\hline LF & $\begin{array}{l}\text { Potência de baixa frequência } \\
\qquad(0,04-0,15 \mathrm{~Hz})\end{array}$ & $\begin{array}{l}\text { Reflete predominantemente } \\
\text { atividade simpática }\end{array}$ \\
\hline $\mathrm{HF}$ & $\begin{array}{l}\text { Potência de alta frequência } \\
\qquad(0,15-0,4 \mathrm{~Hz})\end{array}$ & $\begin{array}{l}\text { Reflete predominantemente } \\
\text { atividade simpática }\end{array}$ \\
\hline $\mathrm{LH} / \mathrm{HF}$ & $\begin{array}{l}\text { Relação de potências de } \\
\text { baixa e alta frequência }\end{array}$ & $\begin{array}{l}\text { Medida do equilíbrio } \\
\text { simpático-vagal }\end{array}$ \\
\hline PT & Poder Total & \\
\hline \multicolumn{3}{|c|}{ Parâmetros da Plotagem de Poincaré } \\
\hline SD1 & $\begin{array}{l}\text { Desvio padrão da largura do } \\
\text { gráfico de Poincaré }\end{array}$ & $\begin{array}{l}\text { Reflete atividade } \\
\text { parassimpática }\end{array}$ \\
\hline SD2 & $\begin{array}{l}\text { Desvio padrão do } \\
\text { comprimento do gráfico de } \\
\text { Poincaré }\end{array}$ & $\begin{array}{l}\text { Reflete a variabilidade global } \\
\text { dos intervalos } R-R\end{array}$ \\
\hline
\end{tabular}

Fonte: Costa AFO, et al., 2021.

\section{RESULTADOS}

\section{Identificação dos estudos}

Foram encontrados 1530 artigos no Google Acadêmico, 119 no Pubmed, 28 no Cochrane e 7 no LILACS, perfazendo um total de 1684 artigos, após a análise de títulos, 79 artigos foram incluídos na análise, com posterior exclusão de 7 duplicatas. Em seguida, durante a leitura integral dos 72 artigos selecionados, 27 artigos foram selecionados. Por fim, a presente revisão de literatura foi realizada com os 8 artigos selecionados (Figura 1). 
Figura 1 - Processo de seleção dos artigos.

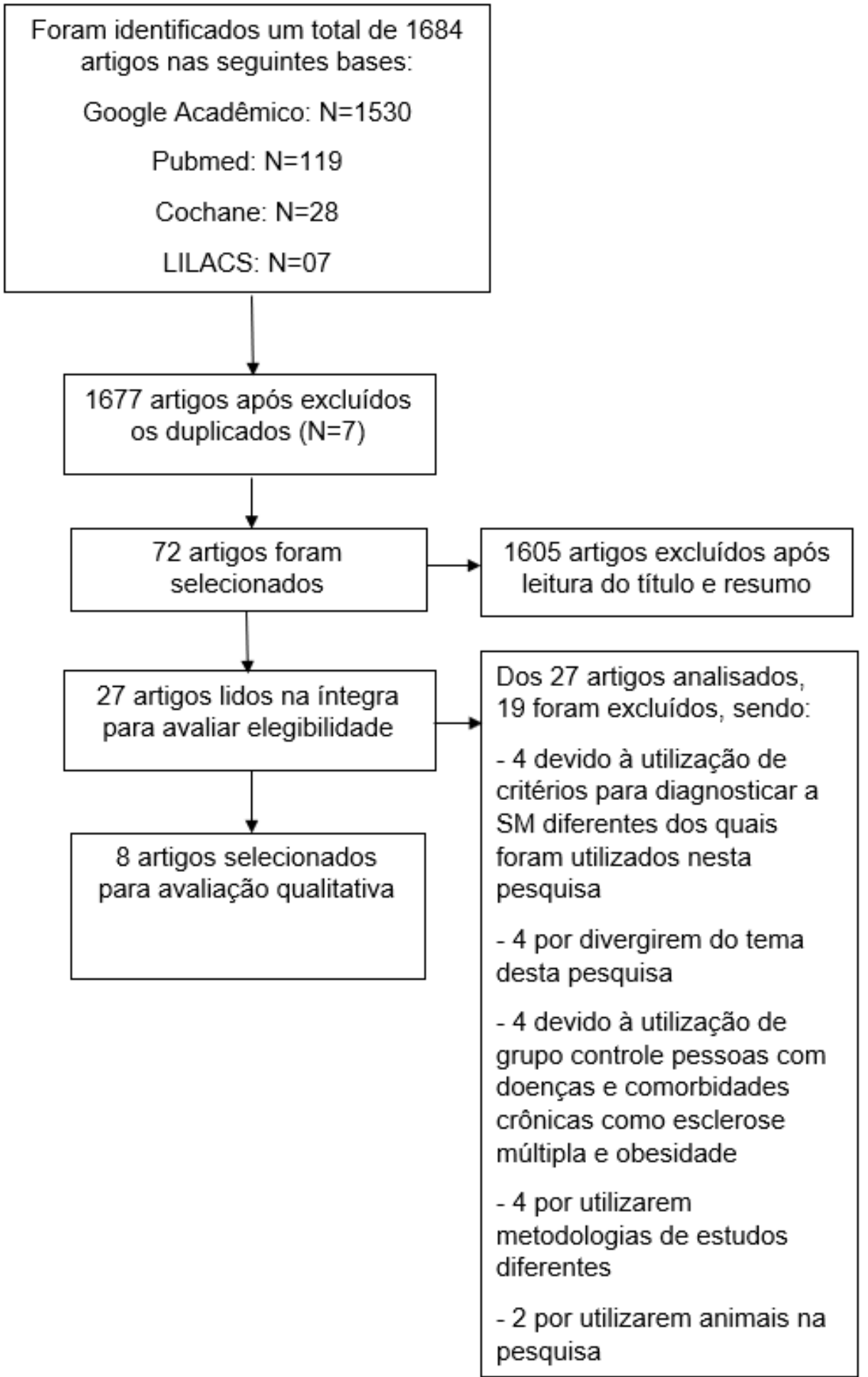

Fonte: Costa AFO, et al., 2021.

\section{Resumo dos estudos incluídos}

Todos os artigos trabalharam com pessoas provindas da população geral, desde adultos jovens a idosos, sendo o participante de menor idade com 18 anos e a maior idade 79 anos. Apenas dois estudos ajustaram a análise por sexo (KANGAS P, et al., 2019; STUCKEY MI, et al., 2015). Sabe-se também que a FC interfere na VFC, porém apenas três estudos incluíram um ajuste para essa variável (KANGAS P, et al., 2019; RASICMILUTINOVIC ZR, et al., 2010; STUCKEY MI, et al., 2015). 
A maior parte dos estudos analisou a VFC a curto prazo durante um período de 5 minutos (KANGAS $P$, et al., 2019; KUBIČKOVÁ A, et al., 2016; MACAGNAN FE, et al., 2019; SAITO I, et al., 2017; STUCKEY MI, et al., 2015). Apenas um estudo fez a análise pelo período de 10 minutos (RODRIGUES JAL, et al., 2017). O registro do eletrocardiograma foi coletado na posição supina Stuckey Ml, et al. (2015), em posição supina e em seguida ortostase por Kangas P, et al. (2019) e Kubičková A, et al. (2016), ou em posição supina e em seguida teste de esforço (MACAGNAN FE, et al., 2019; RODRIGUES JAL, et al., 2017). Saito I, et al. (2017) fez a avaliação através de um sensor de onda de pulso na ponta dos dedos durante 5 min. Finalmente, dois estudos analisaram a VFC através de Holter $24 \mathrm{H}$, Ma Y, et al. (2017) em condições de vida livre e RasicMilutinovic ZR, et al. (2010) em condições mais estáveis de laboratório (Quadro 2).

Quadro 2 - Resumo das características dos artigos incluídos.

\begin{tabular}{|c|c|c|c|c|c|c|c|}
\hline Autores & $\mathbf{N}$ & $\begin{array}{l}\text { Tipo de } \\
\text { Estudo }\end{array}$ & Idade & País & População & $\begin{array}{c}\text { Critérios } \\
\text { diagnósticos } \\
\text { da SM }\end{array}$ & $\begin{array}{l}\text { Critérios } \\
\text { de Homa }\end{array}$ \\
\hline $\begin{array}{l}\text { Ma Y, et al. } \\
\quad(2017)\end{array}$ & 401 & $\begin{array}{c}\text { Caso } \\
\text { Controle } \\
\text { transversal }\end{array}$ & $\begin{array}{l}\text { Acima } \\
\text { de } 20 \\
\text { anos }\end{array}$ & Taiwan & $\begin{array}{l}\text { Homens e } \\
\text { Mulheres }\end{array}$ & $\begin{array}{c}\text { ATP3 com } \\
\text { modificação } \\
\text { da } \\
\text { circunferência } \\
\text { para asiáticos }\end{array}$ & Não \\
\hline $\begin{array}{c}\text { Rasic- } \\
\text { Milutinovic ZR, } \\
\text { et al. (2010) }\end{array}$ & 32 & $\begin{array}{c}\text { Caso } \\
\text { Controle } \\
\text { transversal }\end{array}$ & $\begin{array}{l}\text { Acima } \\
\text { de } 18 \\
\text { anos }\end{array}$ & Sérvia & $\begin{array}{l}\text { Homens e } \\
\text { Mulheres }\end{array}$ & ATP3 & Não \\
\hline $\begin{array}{l}\text { Stuckey Ml, et } \\
\text { al. (2015) }\end{array}$ & 220 & $\begin{array}{c}\text { Caso } \\
\text { Controle } \\
\text { transversal }\end{array}$ & $\begin{array}{l}18-70 \\
\text { anos }\end{array}$ & Canadá & $\begin{array}{l}\text { Homens e } \\
\text { Mulheres }\end{array}$ & ATP3 & Sim \\
\hline $\begin{array}{l}\text { Kubičková A, } \\
\text { et al. (2016) }\end{array}$ & 64 & $\begin{array}{c}\text { Caso } \\
\text { Controle } \\
\text { transversal }\end{array}$ & $\begin{array}{l}24-57 \\
\text { anos }\end{array}$ & $\begin{array}{l}\text { República } \\
\text { Tcheca }\end{array}$ & $\begin{array}{l}\text { Homens e } \\
\text { Mulheres }\end{array}$ & ATP3 & Não \\
\hline $\begin{array}{l}\text { Kangas } P \text {, et } \\
\text { al. }(2019)\end{array}$ & 501 & $\begin{array}{c}\text { Caso } \\
\text { Controle } \\
\text { transversal }\end{array}$ & $\begin{array}{l}19-72 \\
\text { anos }\end{array}$ & Finlândia & $\begin{array}{l}\text { Homens e } \\
\text { Mulheres }\end{array}$ & IDF 2009 & Não \\
\hline $\begin{array}{c}\text { Macagnan FE, } \\
(2018)\end{array}$ & 14 & $\begin{array}{c}\text { Caso } \\
\text { Controle } \\
\text { transversal }\end{array}$ & $\begin{array}{l}18-65 \\
\text { anos }\end{array}$ & Brasil & $\begin{array}{l}\text { Homens e } \\
\text { Mulheres }\end{array}$ & ATP3 & Sim \\
\hline $\begin{array}{c}\text { Rodrigues JAL, } \\
\text { et al. (2017) }\end{array}$ & 119 & $\begin{array}{c}\text { Caso } \\
\text { Controle } \\
\text { transversal }\end{array}$ & $\begin{array}{l}\text { Acima } \\
\text { de } 18 \\
\text { anos }\end{array}$ & Brasil & $\begin{array}{l}\text { Homens e } \\
\text { Mulheres }\end{array}$ & IDF & Não \\
\hline $\begin{array}{l}\text { Saito I, et al. } \\
\quad(2017)\end{array}$ & 2016 & $\begin{array}{c}\text { Caso } \\
\text { Controle } \\
\text { transversal }\end{array}$ & $\begin{array}{l}30-79 \\
\text { anos }\end{array}$ & Japão & $\begin{array}{l}\text { Homens e } \\
\text { Mulheres }\end{array}$ & $\begin{array}{c}\text { ATP3 com } \\
\text { modificação } \\
\text { da } \\
\text { circunferência } \\
\text { para asiáticos }\end{array}$ & Sim \\
\hline
\end{tabular}

Legenda: IDF: International Diabetes Federation; ATP3: National Cholesterol Education Program's Adult Treatment Panel III.

Fonte: Costa AFO, et al., 2021.

\section{FC em pacientes com SM}

Dois estudos não relataram dados sobre a FC (KUBIČKOVÁ A, et al., 2016; SAITO I, et al., 2017). No entanto, Kangas P, et al. (2019), Ma Y, et al. (2017), Macagnan FE, (2018), Rasic-Milutinovic ZR, et al. (2010), Rodrigues JAL, et al. (2017) e Stuckey MI, et al. (2015) realizaram a análise da FC. Desses, quatro constataram um aumento dela em relação aos pacientes SM- (MA Y, et al., 2017; MACAGNAN FE, et al., 2019; RASIC-MILUTINOVIC ZR, et al., 2010; STUCKEY MI, et al., 2015). Porém os achados de Stuckey MI, et al. (2015) foram somente para mulheres e os de Macagnan FE, et al. (2019) somente após o exercício, sendo durante o repouso a FC normal (Quadro 3). 
Quadro 3 - FC em pacientes SM+ em relação a pacientes SM-.

\begin{tabular}{|c|c|c|c|c|}
\hline Autores & Observação & FC & SDNN & RMSSD \\
\hline $\begin{array}{c}\text { Ma Y, et al. } \\
(2017)\end{array}$ & Avaliação de VFC & Aumento & Queda & NS \\
\hline $\begin{array}{c}\text { Rasic-Milutinovic } \\
\text { ZR, et al. (2010) }\end{array}$ & Avaliação de VFC & Aumento & NS \\
\hline $\begin{array}{c}\text { Stuckey Ml, et al. } \\
(2015)\end{array}$ & Mulheres & Aumento & Queda & NS \\
\cline { 2 - 5 } & Homens & NS & NS & NS \\
\hline $\begin{array}{c}\text { Kubičková A, et } \\
\text { al. (2016) }\end{array}$ & Inclinação & Não relatado & Queda & NS \\
\cline { 2 - 5 } & Mulheres - Supino & NS & Não relatado & Não relatado \\
\cline { 2 - 5 } $\begin{array}{c}\text { Kangas P, et al. } \\
(2019)\end{array}$ & Mulheres - Vertical & NS & Não relatado & Não relatado \\
\cline { 2 - 5 } & Homens - Supino & NS & Não relatado & Não relatado \\
\cline { 2 - 5 } & Homens - Vertical & NS & Não relatado & Não relatado \\
\hline \multirow{2}{*}{$\begin{array}{c}\text { Macagnan FE, } \\
(2018)\end{array}$} & Após exercício & Aumento & Queda & Não relatado \\
\cline { 2 - 5 } & Após recuperação & NS & NS & Não relatado \\
\hline $\begin{array}{c}\text { Rodrigues JAL, et } \\
\text { al. (2017) }\end{array}$ & Avaliação de VFC & NS & Não relatado & Não relatado \\
\hline $\begin{array}{c}\text { Saito l, et al. } \\
(2017)\end{array}$ & Avaliação de VFC & Não relatado & NS & Queda \\
\hline
\end{tabular}

Legenda: NS: não significativo; SM+: pacientes com síndrome metabólica; SM-: pacientes sem síndrome metabólica. SDNN: Desvio padrão dos intervalos R-R normais; RMSSD: Raiz quadrada média das diferenças sucessivas. Fonte: Costa AFO, et al., 2021.

\section{Parâmetros do Domínio do Tempo em pacientes com SM SDNN}

Os achados em relação ao SDNN demonstraram-se em parte inconsistentes. Porém, em todos os estudos observou-se uma diminuição deste. Quatro estudos demonstram uma queda do SDNN em relação ao grupo controle (KUBIČKOVÁ A, et al., 2016; MA Y, et al., 2017; MACAGNAN FE, et al., 2019; STUCKEY MI, et al., 2015). Destes citados, apenas um constatou que houve uma forte correlação entre o SDNN e os indivíduos com SM+ (MA Y, et al., 2017). Já os outros dois não encontraram essa correlação evidenciada visto que Stuckey $\mathrm{Ml}$, et al. (2015) percebeu essa alteração somente em mulheres e o outro somente em posição supina (KUBIČKOVÁ A, et al., 2016). Entretanto, deve-se observar que estes estabeleceram a análise através de testes de 5 min, enquanto Ma Y, et al. (2017) fez uma análise de 24h. Para Saito I, et al. (2017), em um primeiro momento houve diferença, porém após retirar os fatores de confusão esse achado não foi mais possível

\section{RMSSD}

Houve um consenso entre os estudos que fizeram a análise do RMSSD de que os pacientes com SM apresentavam uma queda nesse parâmetro. Assim, mostra-se uma consistência na relação da SM com o RMSSD. Kubičková A, et al. (2016) e Saito I, et al. (2017) encontraram uma queda significativa do RMSSD , 
sendo que ambos foram realizados em uma amostra da população geral em uma análise de 5 min ). Saito I, et al. (2017) constatou uma forte relação de RMSSD com a SM, mesmo após a correção de possíveis fatores de confusão como tabagismo, alcoolismo, atividade física e resistência insulínica. Três estudos não encontraram diferenças significativas entre o RMSSD dos pacientes com SM e dos pacientes do grupo controle (MA Y, et al., 2017; RASIC-MILUTINOVIC ZR, et al., 2010; STUCKEY MI, et al., 2015).

\section{Parâmetros do Domínio da Frequência em pacientes com SM}

Resultados encontrados após um teste de esforço são diferentes daqueles encontrados em repouso e isso decorre do fato de a atividade física promover um estímulo autonômico.

\section{VLF}

O VLF está relacionado com a resposta das estruturas suprassegmentais para a regulação da VFC. Por esse motivo, apenas dois estudos levaram esse parâmetro em consideração na pesquisa, constatando uma redução significante do mesmo em relação ao grupo controle (MA Y, et al., 2017; STUCKEY MI, et al., 2015).

\section{LF}

Foram quatro estudos que avaliaram o paciente em posição supina ou ortostase (condições normais) (KANGAS P, et al., 2019; MA Y, et al., 2017; SAITO I, et al., 2017; STUCKEY MI, et al., 2015). Desses, três encontram uma redução do LF, porém possuem algumas individualidades que devem ser observadas. Um deles notou uma redução da LF independentemente da quantidade de componentes da SM presentes (MA Y, et al., 2017). Já para Stuckey MI, et al. (2015) a redução foi encontrada apenas em mulheres. No terceiro Kangas $P$, et al. (2019) a redução foi variável de acordo com o sexo e com a posição, sendo que os homens demonstraram redução com significância tanto em posição supina quanto vertical enquanto as mulheres apresentaram diferenças apenas na posição vertical. Por fim, para Saito I, et al. (2017), não houve alteração após correção da resistência insulínica.

\section{HF}

Todos os estudos que analisaram o componente HF demonstraram uma queda do seu valor nos pacientes com SM + em relação aos SM- o que demonstra a relação estrita e consistente entre HF e SM (KANGAS P, et al., 2019; MA Y, et al., 2017; MACAGNAN FE, et al., 2019; RASIC-MILUTINOVIC ZR, et al., 2010; SAITO I, et al., 2017). De acordo com Ma Y, et al. (2017) essa correlação é independente ao número de componentes da SM encontrados. Vale salientar que ambos são estudos com grande amostra populacional, o que contribui para ratificar esse achado. Kangas $P$, et al. (2019), da mesma forma, mantém a linha de evidências ao encontrar um HF reduzido tanto em posição supina quanto na posição vertical independente do sexo. Apenas um estudo não relatou HF, pois foi realizado cálculos de Poincaré no qual transformava o mesmo em outra variável (KUBIČKOVÁ A, et al., 2016).

\section{LF/HF}

Apenas dois estudos relataram um aumento da relação LF/HF (RASIC-MILUTINOVIC ZR, et al., 2010; SAITO I, et al., 2017). Para três outros estudos não houveram alterações significativas entre o grupo em análise e o grupo controle com relação a proporção LF/HF (KANGAS P, et al., 2019; STUCKEY MI, et al., 2015). Os outros dois estudos não relataram sobre LF/HF (KUBIČKOVÁ A, et al., 2016; RODRIGUES JAL, et al., 2017). Rodrigues JAL, et al. (2017) devido à achados não significativos de LF e HF em separado, acarretou uma relação LF/HF de análise também não significativa e Kubičková A, et al. (2016) por usar o método de Poincaré que utiliza parâmetros diferentes.

\section{PT}

Apenas um estudo fez análise de PT de forma isolada (KANGAS P, et al., 2019). Este encontrou um PT menor tanto no sexo masculino quanto no sexo feminino e tanto no teste em posição supina quanto no teste em posição vertical (Quadro 4). 
Quadro 4 - SM+ em relação a SM- quanto aos parâmetros domínio do frequência.

\begin{tabular}{|c|c|c|c|c|c|c|}
\hline Autores & Observação & VLF & LF & HF & LF/HF & PT \\
\hline Ma Y, et al. (2017) & Análise da VFC & Queda & Queda & Queda & NS & $\begin{array}{l}\text { Não } \\
\text { relatado }\end{array}$ \\
\hline $\begin{array}{l}\text { Rasic-Milutinovic ZR, } \\
\text { et al. (2010) }\end{array}$ & Análise da VFC & NS & $\begin{array}{l}\text { Não } \\
\text { relatado }\end{array}$ & Queda & Aumento & $\begin{array}{l}\text { Não } \\
\text { relatado }\end{array}$ \\
\hline \multirow{2}{*}{$\begin{array}{l}\text { Stuckey Ml, et al. } \\
\qquad(2015)\end{array}$} & Mulheres & Queda & Queda & NS & NS & $\begin{array}{l}\text { Não } \\
\text { relatado }\end{array}$ \\
\hline & Homens & NS & NS & NS & NS & $\begin{array}{l}\text { Não } \\
\text { relatado }\end{array}$ \\
\hline \multirow{2}{*}{$\begin{array}{c}\text { Kubičková A, et al. } \\
\qquad(2016)\end{array}$} & Supino & Não relatado & $\begin{array}{c}\text { Não } \\
\text { relatado }\end{array}$ & $\begin{array}{c}\text { Não } \\
\text { relatado }\end{array}$ & $\begin{array}{c}\text { Não } \\
\text { relatado }\end{array}$ & $\begin{array}{l}\text { Não } \\
\text { relatado }\end{array}$ \\
\hline & Inclinação & Não relatado & $\begin{array}{c}\text { Não } \\
\text { relatado }\end{array}$ & $\begin{array}{c}\text { Não } \\
\text { relatado }\end{array}$ & $\begin{array}{c}\text { Não } \\
\text { relatado }\end{array}$ & $\begin{array}{l}\text { Não } \\
\text { relatado }\end{array}$ \\
\hline \multirow{4}{*}{$\begin{array}{c}\text { Kangas } P \text {, et al. } \\
(2019)\end{array}$} & Mulheres - Supino & Não relatado & NS & NS & NS & Queda \\
\hline & Mulheres - Vertical & Não relatado & Queda & Queda & NS & Queda \\
\hline & Homens - Supino & Não relatado & Queda & Queda & NS & Queda \\
\hline & Homens - Vertical & Não relatado & Queda & Queda & NS & Queda \\
\hline \multirow{2}{*}{ Macagnan FE, (2018) } & Após exercício & Não relatado & Aumento & Queda & Aumento & $\begin{array}{l}\text { Não } \\
\text { relatado }\end{array}$ \\
\hline & Após recuperação & Não relatado & NS & Queda & Queda & $\begin{array}{l}\text { Não } \\
\text { relatado }\end{array}$ \\
\hline $\begin{array}{l}\text { Rodrigues JAL, et al. } \\
\qquad(2017)\end{array}$ & Análise da VFC & Não relatado & NS & NS & $\begin{array}{l}\text { Não } \\
\text { relatado }\end{array}$ & $\begin{array}{l}\text { Não } \\
\text { relatado }\end{array}$ \\
\hline Saito I, et al. (2017) & Análise da VFC & Não relatado & NS & Queda & Queda & $\begin{array}{l}\text { Não } \\
\text { relatado }\end{array}$ \\
\hline
\end{tabular}

Legenda: SM+: pacientes com síndrome metabólica; SM-: pacientes sem síndrome metabólica; NS: não significativo; VLF: potência de frequência muito baixa; LF: potência de baixa frequência; HF: potência de alta frequência; LF/HF: relação de potências de baixa e alta frequência; PT: poder total.

Fonte: Costa AFO, et al., 2021.

\section{Parâmetros de Plotagem de Poincaré}

Apenas três estudos se valeram do cálculo de Poincaré e seus parâmetros SD1 e SD2 em sua metodologia e análise, visto que é uma rede muito específica (KUBIČKOVÁ A, et al., 2016; MA Y, et al., 2017; STUCKEY $\mathrm{Ml}$, et al., 2015). Dois fizeram a coleta de dados através de eletrocardiograma de $5 \mathrm{~min}$ em posição supina, Kubičková A, et al. (2016) e Ma Y, et al. (2017), sendo que Kubičková A, et al. (2016) também fez a análise também em inclinação. Ma Y, et al. (2017) utilizou Holter 24h.

Com relação a SD1, para Stuckey MI, et al. (2015) não houve diferenças relevantes, enquanto para Kubičková A, et al. (2016) houve uma redução significativa na posição supina. Já para Ma Y, et al. (2017) só pode ser observado alterações significativas quando os indivíduos apresentaram 5 componentes de SM. Dado isso, percebe-se que SD1 tem baixa sensibilidade para SM. Em relação a SD2, Ma Y, et al. (2017) percebeu uma maior sensibilidade, estando presente alterações apenas com 3 componentes da SM, ao apresentar valores significativamente menores em relação a indivíduos que tinham 1 ou 2 componentes da síndrome, mas não tinham o quadro sindrômico (Quadro 5). 
Quadro 5 - Parâmetros de Poincaré em SM+ em relação à SM-.

\begin{tabular}{|c|c|c|c|}
\hline Autores & Observação & SD1 & SD2 \\
\hline Ma Y, et al. (2017) & Avaliação da VFC & NS & Queda \\
\hline Rasic-Milutinovic ZR, et al. & Avaliação da VFC & Não relatado & Não relatado \\
\hline \multirow{2}{*}{ Stuckey MI, et al. (2015) } & Mulheres & NS & Queda \\
\cline { 2 - 4 } & Homens & NS & NS \\
\hline \multirow{2}{*}{ Kubíčková A, et al. (2016) } & Supino & Queda & Queda \\
\cline { 2 - 4 } & Inclinação & NS & Queda \\
\hline \multirow{3}{*}{ Kangas P, et al. (2019) } & Mulheres - Supino & Não relatado & Não relatado \\
\cline { 2 - 4 } & Mulheres - Vertical & Não relatado & Não relatado \\
\cline { 2 - 4 } & Homens - Supino & Não relatado & Não relatado \\
\cline { 2 - 4 } & Homens - Vertical & Não relatado & Não relatado \\
\hline \multirow{2}{*}{ Macagnan FE, (2018) } & Após exercício & Não relatado & Não relatado \\
\cline { 2 - 4 } & Após recuperação & Não relatado & Não relatado \\
\hline Rodrigues JAL, et al. (2017) & Análise da VFC & Não relatado & Não relatado \\
\hline Saito I, et al. (2017) & Análise da VFC & Não relatado & Não relatado \\
\hline
\end{tabular}

Legenda: SM+: pacientes com síndrome metabólica; SM-: pacientes sem síndrome metabólica; NS: não significativo; SD1: desvio padrão da largura do gráfico de Poincaré; SD2: desvio padrão do comprimento do gráfico de Poincaré. Fonte: Costa AFO, et al., 2021.

\section{VFC e resistência insulínica}

Quatro estudos analisaram a real influência da resistência insulínica (RI), um fator determinante na patogênese da SM, sobre a VFC, ponderando se a RI era um fator predisponente ou apenas um agravante da disautonomia cardíaca (MACAGNAN FE, et al., 2019; RASIC-MILUTINOVIC ZR, et al., 2010; SAITO I, et al., 2017; STUCKEY MI, et al., 2015). Três estudos se valeram do Homeostatic Model Assessment (HOMA), que é um método utilizado para quantificar a resistência insulínica, na sua análise da populacional (MACAGNAN FE, et al., 2019; SAITO I, et al., 2017; STUCKEY MI, et al., 2015). Para Macagnan FE, et al. (2019) os pacientes SM+ apresentaram RI muito maior em relação aos SM-. Já para Stuckey MI, et al. (2015) essa relação foi significante somente nas mulheres $\mathrm{SM}+\mathrm{e}$ isso ratifica que seus resultados não podem sustentar hipóteses prévias de que a resistência à insulina seja um mecanismo envolvido em alterações na VFC em SM. Em contrapartida, Saito I, et al. (2017), encontrou fortes relações da RI em pacientes SM+ em ambos os sexos. Relatou também que uma VFC baixa contribui para pacientes com SM desenvolverem resistência insulínica. Já Rasic-Milutinovic ZR, et al. (2010) fez sua análise baseado no fato de um indivíduo em estudo ter ou não DM2. Constatou-se que a DM2 é um fator agravante dos parâmetros da VFC, porém percebeu-se que uma queda na VFC é encontrada mesmo nos pacientes SM+ não diabéticos, ou seja, a disautonomia cardíaca precederia o DM2, achado este que está em consonância aos achados de Macagnan FE, et al. (2019) e Saito I, et al. (2017).

\section{DISCUSSÃO}

Foi observado que a VFC é reduzida em SM+ em comparação aos SM-, sendo que essa característica é agravada quanto maior o número de fatores da SM o indivíduo obtiver e quanto mais crônica for a doença (RODRIGUES JAL, et al., 2017; SAITO I, et al., 2017; STUCKEY MI, et al., 2015). Esses achados estão de acordo com estudos previamente publicados e relevantes (CAMBRI LT, et al., 2008; FREITAS ED DE, et al., 2008; STUCKEY MI, et al., 2014). Notou-se, também, que essa redução é mais evidente em mulheres em detrimento aos homens (KANGAS P, et al., 2019; STUCKEY MI, et al., 2014).

Determinada essa redução da VFC procede-se a análise das bandas que a compõem identificando as de maior correlação com a SM. As bandas da frequência refletem os componentes do sistema nervoso autônomo (SNA) o que permite associações de fatores risco cardiometabólicos com os mecanismos autonômicos cardíacos (LIAO D, et al., 1997; THAYER JF, et al., 2010). Averiguou-se que os parâmetros diretamente associados ao sistema parassimpático, RMSSD e HF, possuem maior especificidade com a SM (KANGAS P, 
et al., 2019; KUBIČKOVÁ A, et al., 2016; MA Y, et al., 2017; SAITO I, et al., 2017). Tal característica é explicada devido ao fato desses dois parâmetros permanecerem imutáveis em seus achados, mesmo após exclusão de possíveis fatores de confusão tais como de tabagismo, consumo de álcool, atividade física e resistência insulínica (SAITO I, et al., 2017). Além disso, os achados de diminuição no RMSSD e no HF foram unanimidade em todos os estudos analisados não havendo, portanto, controversas. Dessa forma, conclui-se que essa redução do parassimpático seria o componente responsável pelo desequilíbrio simpatovagal, e pela disautonomia nos indivíduos portadores de SM.

O Plot de Poincaré é um método geométrico para a análise dinâmica da VFC, ou seja, ele permite fazer uma análise não linear da VFC, e estabelece dois parâmetros a serem avaliados: SD1 e SD2 (VANDERLEI LCM, et al., 2009). O SD1 é um índice de registro instantâneo da variabilidade batimento-a-batimento e representa a atividade parassimpática, enquanto o índice SD2 representa a VFC, em registros de longa duração e reflete a variabilidade global (VANDERLEI LCM, et al., 2009). Os três estudos que utilizaram esse método encontram uma relação direta da SM com SD2, o qual se apresentava sempre diminuído em pacientes $\mathrm{SM}+$, representando, portando, uma VFC reduzida. Já para SD1 os achados só foram consistentes quando o paciente apresentava mais de cinco características da SM ou em teste de provocação do SNA. Nesses casos específicos, o que se encontrou em relação ao SD1 era uma redução em relação ao grupo controle, ou seja, uma redução do parassimpático (KUBIČKOVÁ A, et al., 2016; MA Y, et al., 2017; STUCKEY MI, et al., 2015).

Vale enfatizar que SDNN, VLF, LF, LF/HF, PT também podem ser usados na correlação com SM e na maioria das vezes estão alterados em pacientes $\mathrm{SM}$. Assim como a $\mathrm{FC}$, uma vez que ela possui uma relação direta com a VFC de aspecto matemático inverso, ou seja, uma vez que a medida que a FC aumenta há uma diminuição da VFC (STUCKEY MI, et al., 2015) .

Um dos fatores de confusão e que gera dúvidas na relação entre a redução da VFC e a SM é a resistência insulínica (RI). A RI está diretamente associada a dois dos cinco critérios que definem a SM: a obesidade central e o aumento da glicose plasmática, além de fazer parte diretamente da patogênese dessa síndrome (SANTOS CE, et al., 2009). Devido a esses fatos, muitos estudos não chegam a um consenso se a disautonomia cardíaca em pacientes $\mathrm{SM}+$ seria um preditor para a RI ou apenas uma consequência desta. Nesta revisão destacou-se o estudo de Saito I, et al. (2017) por este ter feito seu estudo com um N de 2016 pessoas, número de participantes consideravelmente significativo, e ter levado em consideração na sua pesquisa os critérios de HOMA na avaliação dos voluntários. Dessa forma, através de cálculos matemáticos, Saito I, et al. (2017) conseguiu estabelecer quais parâmetros da VFC sofriam interferência direta ou não da RI. Destarte, o autor chegou à conclusão de que baixo RMSSD, baixo HF e alto LF/HF, foram significativamente associados à SM independente da RI.

\section{CONSIDERAÇÕES FINAIS}

A VFC em pacientes com SM+ encontra-se diminuída, sendo que tal redução mais evidente quando presente em mulheres portadoras da síndrome e mais acentuada quanto mais crônica a doença for e quanto mais critérios diagnósticos de SM o paciente apresentar. Além disso, em relação aos parâmetros avaliados na disautonomia cardíaca, os relacionados à atividade do parassimpático são mais sensíveis do que os outros, sendo eles o RMSSD, HF e SD2, encontrados em todos os artigos de forma reduzida. E por fim, ressalta-se a existência da associação entre a resistência insulínica com a piora da VFC, indicando também a necessidade de novos estudos que expliquem a verdadeira correlação existente entre elas.

\section{REFERÊNCIAS}

1. ALBERTI KGMM, et al. Harmonizing the metabolic syndrome. Circulation, 2009; 120(16):1640-1645.

2. CAMBRI LT, et al. Limiar de variabilidade da frequência cardíaca em teste progressivo de caminhada em diabéticos tipo 2. Revista Brasileira de Ciência e Movimento, 2009; 17(1):1-24

3. CAMBRI LT, et al. Variabilidade da frequência cardíaca e controle metabólico. Arq Sanny Pesq Saúde, 2008; 1(1): 72-82. 
4. CARVALHO MHC, et al. I Diretriz brasileira de diagnóstico e tratamento da síndrome metabólica. 2005; 84(1): 3-28

5. CHANG C-J, et al. Altered cardiac autonomic function may precede insulin resistance in metabolic syndrome. The American journal of medicine, 2010; 123(5): 432-438.

6. ESLER M, et al. Sympathetic nervous system and insulin resistance: from obesity to diabetes. American journal of hypertension, 2001; 14(S7): 304S-309S.

7. FREITAS ED DE, et al. Síndrome metabólica: uma revisão dos critérios de diagnóstico. Revista mineira de enfermagem, 2008; 12(3): 403-411.

8. KANGAS P, et al. Metabolic syndrome is associated with decreased heart rate variability in a sex-dependent manner: a comparison between 252 men and 249 women. Clinical physiology and functional imaging, 2019; 39(2): $160-167$.

9. KUBIČKOVÁ A, et al. Heart rate variability analysed by Poincaré plot in patients with metabolic syndrome. Journal of electrocardiology, 2016; 49(1): 23-28.

10. LAMBERT GW, et al. Sympathetic nervous activation in obesity and the metabolic syndrome-causes, consequences and therapeutic implications. Pharmacology \& therapeutics, 2010; 126(2): 159-172.

11. LEITER LA, et al. Cardiometabolic risk in Canada: a detailed analysis and position paper by the cardiometabolic risk working group. Canadian Journal of Cardiology, 2011; 27(2): e1-e33.

12. LIAO D, et al. Cardiac autonomic function and incident coronary heart disease: a population-based case-cohort study: the ARIC Study. American journal of epidemiology, 1997; 145(8): 696-706.

13. MA Y, et al. Cardiac autonomic alteration and metabolic syndrome: an ambulatory ECG-based study in a general population. Scientific reports, 2017; 7(1): 1-9.

14. MACAGNAN FE, et al. Acute physical effort increases sympathovagal balance responses to autonomic stimulation in metabolic syndrome. Metabolic syndrome and related disorders, 2019; 17(1): 67-74.

15. PONTES LM, et al. Componentes da síndrome metabólica e fatores associados em adolescentes: estudo casocontrole. Rev. AMRIGS, 2016; 60(2): 121-128.

16. RAMOS JS, et al. High-intensity interval training and cardiac autonomic control in individuals with metabolic syndrome: a randomised trial. International journal of cardiology, 2017; 245: 245-252.

17. RASIC-MILUTINOVIC ZR, et al. Do components of metabolic syndrome contribute to cardiac autonomic neuropathy in non-diabetic patients. Saudi Med J, 2010; 31(6): 650-657.

18. RODRIGUES JAL, et al. Caracterização da variabilidade da frequência cardíaca em indivíduos com síndrome metabólica. Revista Brasileira de Medicina do Esporte, 2017; 23(3): 208-212.

19. SAITO I, et al. Low Heart Rate Variability and Sympathetic Dominance Modifies the Association Between Insulin Resistance and Metabolic Syndrome-The Toon Health Study-. Circulation Journal, 2017; 81(10):1447-1453.

20. SANTOS CE, et al. Análise crítica dos critérios da OMS, IDF e NCEP para síndrome metabólica em pacientes portadores de diabetes melito tipo 1. Arquivos Brasileiros de Endocrinologia \& Metabologia, 2009; 53(9): $1096-1102$.

21. SILVA LR, et al. Cardiac autonomic modulation and the kinetics of heart rate responses in the on-and off-transient during exercise in women with metabolic syndrome. Frontiers in physiology, 2017; 8: 542:1-9

22. STUCKEY MI, et al. Associations between heart rate variability, metabolic syndrome risk factors, and insulin resistance. Applied Physiology, Nutrition, and Metabolism, 2015; 40(7): 734-740.

23. STUCKEY MI, et al. Heart rate variability and the metabolic syndrome: a systematic review of the literature. Diabetes/metabolism research and reviews, 2014; 30(8): 784-793.

24. THAYER JF, et al. The relationship of autonomic imbalance, heart rate variability and cardiovascular disease risk factors. International journal of cardiology, 2010; 141(2): 122-131.

25. VANDERLEI LCM, et al. Noções básicas de variabilidade da frequência cardíaca e sua aplicabilidade clínica. Brazilian Journal of Cardiovascular Surgery, 2009; 24(2): 205-217. 